pulmonary arterial hypertension (PAH) and predictive risk factors in a cohort of patients with antiphospholipid antibodies.

Methods: We included 232 patients from our cohort who underwent an echocardiogram. A total of $84(36 \%)$ patients with primary antiphospholipid syndrome (PFS), 47 (20\%) with APS secondary to systemic lupus erythematosus (SLE), 47 (20\%) patients with antiphospholipid antibodies 23\%) with SLE without AAF.

The determinations of AAF and lupus anticoagulant were performed according to the indications of the international thrombosis society.

Statistical analysis was performed with SPSS 18; using the Chi square test and the Fisher exact test.

Results: In patients with AAF, the echocardiogram was pathological in 88 patients $(52 \%)(p=0.023)$. Valvular affectation was evidenced in $64(38 \%)(p=0.005)$ and PAH in $16(p=n s)$. Seventeen patients $(35 \%)$, SAF $(48 \%)$, SAFS $(26 \%)$, AIF silent $(14 \%)$ and 9 patients in the non-AAF group $(12 \%)$ presented with valvular affectation $(\mathrm{p}=0.002)$. PAH presented 19 patients, 9 with SAFP $(47 \%), 6$ in the SAFS group (32\%), 1 in the silent AAF group (5\%) and 3 in the non-AAF group $(16 \%)(p=)$. Both PAH and valvular involvement were asymptomatic in most cases, although two patients required valvular replacement. The most frequently affected valve in all groups was mitral valve $(84 \%)$, except in patients with PAH where the most prevalent valvular pathology was tricuspid insufficiency. Patients with valvulopathy and APS had a higher prevalence of total thrombosis than SAF without valvulopathy $(p=0.05)$. Patients with valvulopathy also significantly increased stroke and thrombocytopenia $(p=0.04)$. Patients with valvulopathy had lupus anticoagulant more frequently $(p=0.04)$, with no difference for the rest of AAF. Conclusions: Subclinical valvular involvement is very common in patients with AAF. Every patient with AAA should be given an echocardiogram in the initial protocol of their study in order to rule out both significant valvulopathy and PAH that can modify the management of the condition.

Disclosure of Interest: None declared

DOI: 10.1136/annrheumdis-2017-eular.2548

\section{SAT0265 CLINICAL CHARACTERISTICS OF SYSTEMIC LUPUS ERYTHREMATOSUS IN AN EGYPTIAN POPULATION: A RETROSPECTIVE COHORT}

B. Medhat ${ }^{1}$, T. El Hadidi ${ }^{2}$, K. El Hadidi ${ }^{1}$, A. Yousri ${ }^{1}$, D. Hassan ${ }^{1}$, F. Talaat ${ }^{1}$, L. Maged ${ }^{1}$, M. Shabaan ${ }^{3}$, M. Eissa ${ }^{1}$, N. Hamdy El Gobashy ${ }^{1}$, N. Sobhy ${ }^{1}$, N. Abd El Baki ${ }^{1}$, R. El Refai ${ }^{1}$, S. Ghoneim ${ }^{1}$, W. AbdRahman ${ }^{1}$. ${ }^{1}$ Rheumatology and Rehabilitation, Faculty of Medicine-Cairo University; ${ }^{2}$ Rheumatology and Rehabilitation, Armed forced hospital; ${ }^{3}$ Rheumatology and Rehabilitation, Helwan hospital, Cairo, Egypt

Background: Systemic lupus erythrematosus (SLE) is an autoimmune disease with a myriad of manifestations, that could vary among different ethnic and racial groups.

Objectives: To study the prevalence of various manifestations of SLE in an Egyptian population.

Methods: Information in this study was derived from the medical records of SLE patients who followed up in a private clinic in Cairo from January 1980 to June 2016.

Results: This descriptive retrospective case series included 1109 juvenile (19.4\%) and adult $(80.6 \%)$ patients, of which $114(10.3 \%)$ were males and 995 were females $(89.7 \%)$. Age of onset showed a mean of $26 \pm 11.19$ years, and the mean of disease duration was $48.78 \pm 58.46$ months (median: 26 years). The most common manifestations were synovitis $(76.7 \%)$, malar rash $(48.5 \%)$, leukopenia $(45.7 \%)$, and photosensitivity (45.6\%). At least one of the antiphospholipid antibodies was present in $41.8 \%$ of the patients tested for APL (636 patients). However thromboembolic manifestations and/or recurrent fetal loss occured in $11.5 \%$ of the patients. Neuropsychiatric manifestations were evident only in $6.4 \%$ of the patients, with seizures being the most common neuropsychiatric manifestation, present in $4 \%$ of the patients. $33.1 \%$ of the patients had nephritis, which followed the onset of the disease by a mean duration of $20 \pm 21.3$ months (median $=12$ months). There were gender differences in the disease characteristics. Cutaneous vasculitis, nephritis, and hypocomplementemia were statistically higher in males $(p=0.012$, $p=0.01$, and $p=0.041$ respectively). Whereas, synovitis, and alopecia were statistically higher in females $(\mathrm{p}=0.012$ and $\mathrm{p}=0.006$ respectively). Patients with juvenile onset had a statisticaly higher frequency of nephritis $(0=0.01)$, seizures $(p=0.012)$ haemolytic anemia $(p=0.001)$, and hypocomplementinemia $(p=0.02)$.

Conclusions: Synovitis and malar rash were the most common manifestations in our study. Secondary antiphospholipid was present in $11.5 \%$ of the patients. Male patients and juvenile patients showed a tendency towards a more severe disease.

Disclosure of Interest: None declared

DOI: 10.1136/annrheumdis-2017-eular.2151

\section{SAT0266 EFFECT OF THE METABOLIC SYNDROME ON ORGAN DAMAGE AND MORTALITY IN CHINESE PATIENTS WITH SYSTEMIC LUPUS ERYTHEMATOSUS: A LONGITUDINAL ANALYSIS}

C.C. Mok, S.M. Tse, L.Y. Ho. Medicine, Tuen Mun Hospital, HK, Hong Kong

Objectives: To study the effect of the metabolic syndrome (MetS) on organ damage and mortality in patients with SLE.

Methods: Consecutive patients who fulfilled $\geq 4$ ACR criteria for SLE and were assessed for the presence of the MetS between 2010 and 2011 were included. Those patients who did not have MetS assessment or succumbed before 2010 were excluded. The MetS was defined by the updated joint consensus criteria, using the Asian criteria for central obesity, when $\geq 3$ of the following components were present: (1) Increased waist circumference to $>90 \mathrm{~cm}$ in men or $\geq 80 \mathrm{~cm}$ in women; (2) Elevated blood pressure to $\geq 130 / 85 \mathrm{mmHg}$ or requiring drug therapy; (3) Elevated serum triglyceride level to $\geq 1.7 \mathrm{mmol} / \mathrm{L}$; (4) Reduced serum high density lipoprotein (HDL)-cholesterol to $<1.0 \mathrm{mmol} / \mathrm{L}$ in men and $\leq 1.3 \mathrm{mmol} / \mathrm{L}$ in women; and (5) Elevated fasting glucose level to $\geq 5.6 \mathrm{mmol} / \mathrm{L}$. Longitudinal data regarding new organ damage, vascular events and mortality on follow-up were retrieved from our cohort database. The association of the MetS with new organ damage and mortality was studied by logistic regression analyses.

Results: 577 SLE patients were studied $(93 \%$ women; age at entry $41.2 \pm 13.4$ years; SLE duration $9.3 \pm 7.2$ years). The mean follow-up time of the patients since entry was $66.3 \pm 1.8$ months. The mean body mass index (BMI) of the patients was $22.3 \pm 3.9 \mathrm{~kg} / \mathrm{m}^{2}\left(11 \%>27 \mathrm{~kg} / \mathrm{m}^{2}\right)$. A total of $85(14.7 \%)$ patients qualified the MetS (28\% fulfilling waist; $20 \%$ fulfilling blood pressure; $25 \%$ fulfilling triglyceride; $33 \%$ fulfilling HDL and $9.2 \%$ fulfilling glucose criteria). New organ damage and vascular (coronary, cerebrovascular and peripheral vascular) events developed in $128(22 \%)$ and $23(4.0 \%)$ patients, respectively. The most common new arterial events were stroke $(50 \%)$, acute coronary syndrome $(33 \%)$ and peripheral vascular disease $(17 \%)$. Thirty-nine $(6.8 \%$ ) patients died (infection 36\%; vascular causes $18 \%$; cancer $15 \%$; lung fibrosis $8 \%$; suicide $3 \%$ ). Patients with the MetS $(\mathrm{N}=85)$, when compared to those without $(\mathrm{N}=492)$, had significantly higher SDI accrual at their last clinic visits $(0.70 \pm 1.0$ vs $0.26 \pm 0.6 ; p<0.001)$. Regarding individual systems, the increase in SDI scores in the ocular, renal, cardiovascular, musculoskeletal and endocrine (new diabetes mellitus) systems were significantly higher in the MetS group of patients. New vascular events ( $11 \%$ vs $2.8 \% ; p=0.001)$, all-cause mortality ( $14 \%$ vs $5.5 \% ; p=0.003$ ), death due to vascular complications ( $7.1 \%$ vs $0.2 \% ; p<0.001)$ were significantly more common in patients with MetS than those without. Logistic regression revealed that the MetS was significantly associated with new damage in the ocular (OR 2.77 [1.05-7.34]; $p=0.04$, renal (OR 4.72 [1.86-12.0]; $p=0.001$ ), cardiovascular (OR 3.66 [1.03-12.9]; $p=0.04]$ and endocrine system (OR 41.9 [4.93-357]; $p=0.001$ ), adjusted for age, sex, SLE duration and the antiphospholipid antibodies (IgG-anticardiolipin or the lupus anticoagulant). The presence of the MetS increased the risk of new vascular events (OR 2.94 [1.18-7.31]; $p=0.02$ ), all-cause mortality (OR 1.60 [0.73-3.47]; $p=0.24$ ) and vascular mortality (OR $30.3[3.42-268] ; p=0.002$ ) after adjustment for the same covariates.

Conclusions: In this 5-year longitudinal study, the MetS is significantly associated with new organ damage, vascular events and mortality in patients with SLE.

Disclosure of Interest: None declared

DOI: 10.1136/annrheumdis-2017-eular.3939

\section{SAT0267 SERUM 25-HYDROXYVITAMIN D3 LEVELS AND FLARES OF SYSTEMIC LUPUS ERYTHEMATOSUS: A LONGITUDINAL COHORT ANALYSIS}

C.C. Mok ${ }^{1}$, R. Singh ${ }^{2}$, L.Y. Ho ${ }^{1}$, P. Jannetto ${ }^{2} .{ }^{1}$ Medicine, Tuen Mun Hospital, HK, Hong Kong; ' 2 Laboratory Medicine and Pathology, Mayo Clinic, Rochester, United States

Objectives: To study the relationship between serum 25-hydroxyvitamin D3 levels and flares of systemic lupus erythematosus (SLE) in a longitudinal cohort of Chinese patients.

Methods: Patients who fulfilled $>4$ of the ACR criteria for SLE were recruited from our rheumatology out-patient clinics in November 2011. Blood was taken at $10 \mathrm{AM}$ and was assayed for the serum levels of 25-hydroxyvitamin D3 by liquid chromatography tandem mass spectrometry (LC-MS/MS). Patients were stratified according to the 25-hydroxyvitamin D3 levels; group $1(<15 \mathrm{ng} / \mathrm{ml}$, deficiency); group 2 (15-30ng/ml, insufficiency); and group 3 (>30 $\mathrm{ng} / \mathrm{ml}$, adequate); and were followed longitudinally every 2-4 months for serial assessment of disease activity (by SELENA-SLEDAI) and the occurrence of mild/moderate or severe SLE flares (by SELENA flare instrument). Comparison was made among these groups in the baseline and mean summated SLEDAI over time (area under the curve), and the annual incidence of mild/moderate and severe flares by the one-way ANOVA test. Results: 276 SLE patients were studied (94\% women; age $41.0 \pm 13.8$ years; SLE duration $8.7 \pm 6.6$ years). $25(9.1 \%)$ patients had eGFR $\leq 60 \mathrm{ml} / \mathrm{min}$. The proportion of patients with 25-hydroxyvitamin D3 levels of $<15,15-30,>30 \mathrm{ng} / \mathrm{ml}$ was $26 \%, 54 \%$ and $20 \%$, respectively. Patients with vitamin D deficiency (group 1 ) were significantly younger, had lower body mass index (BMI) but higher baseline eGFR and SLEDAI scores when compared with the other groups. No significant differences in the clinical manifestations were observed among the three groups of patients except for lower prevalence of facial rash in group $3(p=0.02)$. After a mean follow-up of $32.5 \pm 5.5$ months, 153 mild flares and 91 severe flares developed in our patients. The mean summated SLEDAI score over time was: $3.2 \pm 2.0$ (group 1); $2.4 \pm 1.9$ (group 2 ); and $2.7 \pm 2.1$ (group 3 ), respectively $(p=0.02$ ). The annual incidence of mild/moderate and severe flares was: $0.26 \pm 0.39$ and $0.20 \pm 0.45$ (group 1); $0.20 \pm 0.33$ and $0.09 \pm 0.22$ (group 2); and $0.20 \pm 0.32$ and $0.14 \pm 0.46$ (group 3 ), respectively ( $p=N S$ in all). In a subgroup of 73 patients who did not have clinical or serological SLE activity at baseline (SLEDAI=0), a similar 
but non-significant trend of higher annual rates of mild/moderate and severe flares over time was also observed in patients with vitamin $D$ deficiency. At the last visit, 27 (10\%) patients had new damage scores; 5 patients had new vascular events; and 4 patients had new onset diabetes mellitus. There were no significant differences among the three groups of patients with regard to the incidence of new damage or vascular events over time.

Conclusions: Vitamin D insufficiency and deficiency was frequent in our cohort of SLE patients. Patients with vitamin D deficiency were associated with higher baseline and mean disease activity scores, as well as a tendency of more severe lupus flares over time.

Disclosure of Interest: None declared

DOI: 10.1136/annrheumdis-2017-eular.3945

\section{SAT0268 CLINICAL PRESENTATION OF NASAL INVOLVEMENT IN PRIMARY SJÖGREN'S SYNDROME: A MULTIDISCIPLINARY TEAM APPROACH TO A NEGLECTED AND DISABLING CONDITION}

C. Baldini ${ }^{1}$, V. Seccia ${ }^{2}$, E. Elefante ${ }^{1}$, M. Scarano ${ }^{2}$, L. Cristofani ${ }^{2}$, F. Ferro ${ }^{1}$ N. Luciano ${ }^{1}$, M. Mosca ${ }^{1} .{ }^{1}$ Clinical and Experimental Medicine, Rheumatology Unit, University of Pisa; ${ }^{2}$ Otorhinolaryngology Unit, University of Pisa, Pisa, Italy

Background: Dry nose is reported quite frequently by patients affected by primary Sjögren's syndrome (pSS) in daily practice. However, a clear definition of nasal involvement in pSS is not available.

Objectives: a) to explore clinical presentation of nasal involvement in pSS analyzing key symptoms, objective findings at the inspection of the external and inner nose and nasal cytology b) to investigate any associations/correlation between nasal involvement and other clinical-serological disease manifestations c) to assess the overall impact of nasal involvement on patients reported outcomes (PROs).

Methods: Consecutive pSS patients (AECG 2002) were seen by a team of rheumatologists and ENT specialists. In addition to a standard rheumatologic evaluation, all the patients underwent a complete ENT evaluation. Nasal symptoms (i.e dry nose, nasal stuffiness, crusting, hyposmia) were collected by an "ad hoc questionnaire" and scored by the patients on visual analogique scales. Inspection of the external and inner nose, endoscopy of the nasal cavity and nasal cytology were performed as well. Allergy testing were also carried out when indicated. The following tools were used to assess PROs: ESSPRI, SF-36 and SNOT-22.

Results: Forty-six pSS patients were included in the study [M:F=45:1; median age (IQR):64 (53-70); median disease duration (IQR): 66 months (24-120)]. Nasal symptoms ranged from: dryness in the nose $(56.5 \%)$, crusting 10/46 $(21.7 \%)$, nasal stuffiness 20/46 (45.5\%) and hyposmia $7 / 46(15.2 \%)$. Thirteen patients did not present signs of nasal involvement, whereas 21 patients $(45.7 \%)$ presented rhinitis sicca (RS), $6(13 \%)$ allergic rhinitis (AR), $4(8.7 \%)$ chronic rhinosinusitis (CRS) and $2(4.3 \%)$ non-allergic rhinitis (NAR). Patients with nasal involvement were more frequently seronegative $(p=0.04)$ and presented significantly higher SNOT-22 scores $(p=0.008)$ when compared to patients without nasal involvement; no additional demographic or clinical differences between the two groups. Allergy testing were more frequently positive in patients with RS and AR. Nasal cytology showed that the rates of the cells (eosinophils and neutrophils) in patients without nasal involvement were negligible whereas they were significantly increasead in pSS patients with RS and AR. The SNOT-22 $(r=-.433, p=0.02)$ and the scores assigned to the VAS of nasal dryness $(r=-.755, p=0.003)$ and crusting $(r=-.794$, $\mathrm{p}=0.001$ ) strongly correlated with SF-36 questionnaire. SNOT-22 also correlated with the ESSPRI $(r=.399, p=0.04)$, whereas we did not find a correlation between VAS scores assigned to nasal dryness and VAS scores of oral and ocular dryness. PROs related to nasal symptoms were significantly influenced by a concomitant diagnosis of fibromyalgia.

Conclusions: Rhinitis sicca was the most common clinical presentation of nasal involvement in pSS patients, especially in seronegative patients. Apparently, nasal symptoms correlated weakly with ocular and oral dryness. PROs exploring nasal symptoms revealed that nasal involvement impact significantly on patients quality of life.

Disclosure of Interest: None declared

DOI: 10.1136/annrheumdis-2017-eular.4349

\section{SAT0269 RDW LEVELS ARE ASSOCIATED WITH DAMAGE ACCRUAL IN SYSTEMIC LUPUS ERYTHEMATOSUS PATIENTS}

C. Reategui-Sokolova ${ }^{1}$, M. Ugarte-Gil ${ }^{1}$, R. Gamboa-Cárdenas ${ }^{1}$, F. Zevallos ${ }^{1}$, ${ }_{\text {J. Cucho-Venegas }}{ }^{1}$, J. Alfaro-Lozano ${ }^{1}$, M. Medina $^{1}$, Z. Rodríguez-Bellido ${ }^{1}$, C. Pastor-Asurza ${ }^{1}$, G. Alarcón ${ }^{2}$, R. Perich-Campos ${ }^{1} .{ }^{1}$ Rheumatology, Hospital Guillermo Almenara Irigoyen, Lima, Peru; ${ }^{2}$ School of Medicine, The University of Alabama, Birmingham, United States

Background: Systemic Lupus Erythematosus (SLE) patients show higher Red blood cells Distribution Width (RDW) regardless of anaemia status ${ }^{1}$. RDW has been found to positively correlate with serum IgM, CRP, ESR, and SLE Disease Activity Index 2000 (SLEDAI-2K), and glucocorticoid treatment decreased both SLEDAI-2K and RDW ${ }^{2}$

Objectives: To determine whether RDW levels in SLE are associated with damage accrual.
Methods: This cross-sectional study was conducted in 276 SLE patients, 257 females and 19 males. Evaluations included interview, medical records review, physical examination and laboratory tests. Disease activity was measured with the SLEDAI. Damage accrual was ascertained with the SLICC/ACR damage index (SDI). Univariable and multivariable Poisson regression models were performed to determine if RDW levels were associated with damage accrual. These models were stratified by tertiles of RDW. The multivariable model was adjusted for variables known to be associated with this outcome [age at diagnosis, gender, socioeconomic status, ethnicity, tobacco use, disease duration, SLEDAl, anemia, antimalarials and immunosuppressive drugs use, average daily dose and time of exposure to prednisone (PDN)].

Results: The patients mean (SD) age at diagnosis was 34.38 (13.33) years; nearly all patients were mestizo. Disease duration was $7.04(6.16)$ years. The SLEDAI was $5.24(4.67)$ and the SDI 0.92 (1.28). The average daily dose of PDN was $6.90(6.07) \mathrm{mg} / \mathrm{d}$ and the time of exposure to PDN was $6.58(9.59)$ years. RDW levels were 14.57 (1.52)\%. Hemoglobin levels were $12.4(1.7) \mathrm{g} / \mathrm{dl}$. We divided the RDW levels into tertiles with cut points in 13.8 and 14.0; the highest tertiles were associated with disease damage; with a Rate Ratio (RR) 1.57 (1.07-2.28; p: 0.020 for the highest tertile, and 1.67 (1.15-2.42; p: 0.007) for the medium tertile.

Conclusions: Higher RDW levels are associated with damage accrual in SLE patients independent of other well-known risk factors for such occurrence.

References:

[1] Vayá A, Alis R, Hernández J-L, et al. RDW in patients with systemic lupus erythematosus. Influence of anaemia and inflammatory markers. Clin Hemorheol Microcirc 2013; 54: 333-9.

[2] Hu Z-D, Chen Y, Zhang L, et al. Red blood cell distribution width is a potential index to assess the disease activity of systemic lupus erythematosus. Clin Chim Acta 2013; 425: 202-205.

Disclosure of Interest: None declared

DOI: 10.1136/annrheumdis-2017-eular.6368

\section{SAT0270 ULTRASONOGRAPHY OF MAJOR SALIVARY GLANDS IN JUVENILE SJÖGREN'S SYNDROME - PRELIMINARY FINDINGS IN A MULTI-CENTER STUDY}

D.S. Hammenfors ${ }^{1}$, V. Valim ${ }^{2}$, B. Bica ${ }^{3}$, S.G. Pasoto ${ }^{4}$, V. Lilleby ${ }^{5}$, J.C. Nieto-González ${ }^{6}$, C.A. Silva ${ }^{7}$, E. Mossel ${ }^{8}$, R.M. Pereira ${ }^{9}$, H. $^{\text {Bootsma }}{ }^{10}$ J.G. Brun ${ }^{11}$, R. Jonsson ${ }^{12}$, M.V. Jonsson ${ }^{13} .{ }^{1}$ Department of Rheumatology, Haukeland university hospital, Bergen, Norway; ${ }^{2}$ Department of Rheumatology/Medical Clinic, Federal University of Espirito Santo, Vitoria; ${ }^{3}$ Department of Rheumatology, Federal University of Rio de Janeiro, Rio de Janeiro; ${ }^{4}$ Sjögren's syndrome outpatient/Hospital das Clinicas HCFMUSP, University of São Paulo, São Paulo, Brazil; ${ }^{5}$ Department of Rheumatology, Oslo University Hospital, Oslo, Norway; ${ }^{6}$ Department of Rheumatology, Hospital General Universitario Gregorio Marañón, Madrid, Spain; ${ }^{7}$ Department of Pediatric Rheumatology, Federal University of São Paulo, São Paulo, Brazil; ${ }^{8}$ Department of Rheumatology and Clinical Immunology, University Medical Center Groningen, Groningen, Netherlands; ${ }^{9}$ Rheumatology Division - School of Medicine, University of São Paulo, São Paulo, Brazil; ${ }^{10}$ Department of Rheumatology and Clinical Immunology, University Medical Center Groningen, University of Groningen, Groningen, Netherlands; ${ }^{11}$ Department of Clinical Science - Section for Rheumatology; ${ }^{12}$ Broegelmann Research Laboratory, Department of Clinical Science; ${ }^{13}$ Department of Clinical Dentistry - Section for Oral and Maxillofacial Radiology, University of Bergen, Bergen, Norway

Background: Juvenile Sjögren's syndrome (jSS) is a rare, poorly defined and possibly underdiagnosed condition. There is little information on the use of major salivary gland ultrasonography (SGUS) in this patient-group.

Objectives: To characterize symptoms and clinical findings of jSS and to investigate SGUS as a diagnostic tool.

Methods: Sixty-four patients were recruited from Brazil $(n=40)$, Norway $(n=11)$, the Netherlands $(n=8)$ and Spain $(n=5)$. All patients had disease onset at the age of 18 or younger. Clinical examination and sialometry was performed in 60/64 patients. Additional clinical information was obtained from the medical records and through patient interview. SGUS of the parotid and submandibular glands was performed in all patients using linear high-frequency transducers $(6-15 \mathrm{MHz})$, by an expert in SGUS. Glandular homogeneity and presence of hypoechogenic areas were evaluated and glands characterized as normal or SS-like.

Results: The female:male ratio was 6:1. Mean age at diagnosis was 12.1 years (range 4-18), with first symptoms occurring at 10.3 years (range 1-17). Time from onset of symptoms until diagnosis was 1.6 years (range $-2-8$ years). Subjective oral and ocular symptoms were reported in $70 \%$ and $64 \%$ patients, respectively. Reduced secretion of tears was detected in $41 \%$ patients, and hyposalivation in $31 \%$ patients. Minor salivary gland lip biopsy had been performed and focus score determined in 34 patients; 28 biopsies $(82 \%)$ had focus score $\geq 1$. Serologically, $92 \%$ were positive for ANA, $73 \%$ were anti-Ro/SSA+, 38\% were anti-La/SSB+, and $41 \%$ were RF+. Salivary gland enlargement had been experienced by $53 \%$ of the patients; one patient had also experienced lacrimal gland enlargement. Systemic manifestations at some time-point, was registered in $66 \%$ of the patients. Systemic treatment at inclusion was registered in $67 \%$ of the patients; previous systemic treatment was registered in $83 \%$. Diagnostic criteria for primary Sjögren's syndrome (pSS) was fulfilled by $34 / 64$ patients $(53 \%)$ and $39 / 64$ patients $(61 \%)$, 